\title{
LAS EXPORTACIONES DE MINERALES EN EL PERÚ DE CARA AL BICENTENARIO: ANÁLISIS ECONÓMICO, PROYECCIÓN ESTADÍSTICAY PERSPECTIVAS PARA EL MEDIANO PLAZO
}

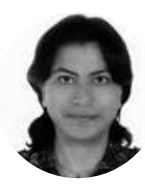

JANNET CANO

- Profesora de Economía Empresarial

Dirección de Administración y Finanzas

Facultad de Negocios, UPC

\section{MANUEL OTÁROLA}

- Profesor de Econometría

Carrera de Ingeniería Económica y de Negocios

Facultad de Ciencias Empresariales, Universidad Científica del Sur, Perú

\section{RESUMEN}

Dada la importancia del sector externo en la evolución de la economía peruana, en este artículo se analizan las características de las exportaciones en el periodo 1950-2016. Asimismo, se comparte un pronóstico de las mismas hasta el año 2021, considerando datos mensuales para el periodo 2009 - 2017, teniendo en cuenta el vínculo de éstas con sus respectivos precios y volúmenes exportados, para el caso de los cuatro principales metales que el Perú vende en el extranjero. Luego de caracterizar las series a partir del análisis de volatilidad y de las pruebas de raíz unitaria, se hicieron pronósticos desde una curva de tendencia. Los resultados auguran tendencias crecientes que deberán contribuir a un mayor valor exportado.

Palabras clave: Sector económico externo, pronósticos económicos, series de tiempo.

\footnotetext{
ABSTRACT

Given the importance of the external sector in the evolution of the Peruvian economy, this paper analyzes export characteristics in the 1950-2016 period. Additionally, using monthly time series data for the 20092017 period, we forecast these exports through 2021, for which we take into account their link their respective prices and volumes, in the case of the four main metals this country exports.
}

After characterizing the series based on volatility analysis and unit root tests, forecasts are made based on a statistical trend curve. The results show growing trends that would contribute to greater export value.

Keywords: Foreign economic sector, economic forecasting, time series.

\begin{abstract}
El Perú se encuentra a poco más de tres años de completar su segundo siglo como república independiente. Con motivo de esa fecha especial, viene bien reflexionar sobre lo que el país ha logrado, sobre todo en años recientes, y acerca de las perspectivas que se avizoran para el mediano plazo. En esta oportunidad, el análisis se concentra en lo ocurrido con las exportaciones, específicamente de los cuatro principales minerales de exportación, dada su importancia dentro de las exportaciones totales y por ser una fuente considerable de divisas. Puntualmente, el objetivo del presente estudio es pronosticar la tendencia de las exportaciones totales a partir de los pronósticos de los cuatro principales productos mineros de exportación: cobre, oro, plomo y zinc.
\end{abstract}

Las proyecciones se realizaron en tres etapas: primero se analizó la volatilidad de los precios internacionales de los minerales seleccionados, luego se aplicó el test aumentado de Dickey-Fuller sobre raíces unitarias (para identificar si las series están integradas a niveles o a primeras diferencias), para finalmente, tomando en cuenta las características seriales, efectuar los pronósticos a partir de la identificación de una curva de tendencia. Considerando las expectativas de los agentes económicos y del Banco Mundial, los pronósticos auguran tendencias crecientes, las cuales permitirían seguir en la senda de un cada vez mayor valor exportado.

\section{ANTECEDENTES: IMPORTANCIA DEL SECTOR EXTERNO EN LA ECONOMÍA}

El intercambio comercial entre países cobra relevancia al permitir mejorar la calidad de vida de sus habitantes mediante el acceso a una mayor diversidad de productos y servicios. Más aún, varios autores a lo largo de la historia — tal es el caso de Smith (1776), Ricardo (1817), Mill (1848), Heckscher (1935), Viner (1937), Haberler (1936), Samuelson (1962), Nurkse (1961), Bhagwati, Panagariya y Srinivasan 
(1998), Deardorff (1984), Feenstra (1998) y Krugman (2000), entre otros- han señalado la promoción, el desarrollo y/o la diversificación de exportaciones como los motores principales del crecimiento económico de una nación. Estudios efectuados en años recientes, como el de Braun, Feldman, Junowicz y Roitman (2008), ponen énfasis en el tipo de bienes o de servicios que se exportan, a la luz de la evidencia de los llamados "tigres del sudeste asiático".

En lo que sigue, se presenta una revisión de las cifras ${ }^{1}$ para el caso peruano durante el periodo 1950-2016.

En primer lugar, en el gráfico 1 se muestran los datos del PBI real. Allí destacan hitos importantes, como el estancamiento durante la década de 1980 y el crecimiento, con ligeros altibajos, de los últimos 27 años, los que se hacen más notorios con las cifras por habitante, compartidas en el gráfico 2 .

\section{Gráfico 1. Perú: PBI total, 1950-2016} (en millones de soles de 2007)

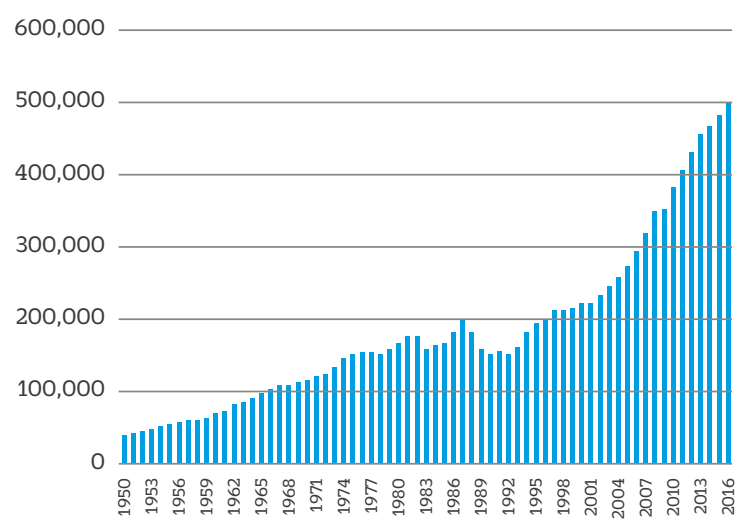

Fuente: BCRP e INEI.

Elaboración propia.

\section{Gráfico 2. Perú: PBI per Cápita, 1950-2016} (en soles de 2007)

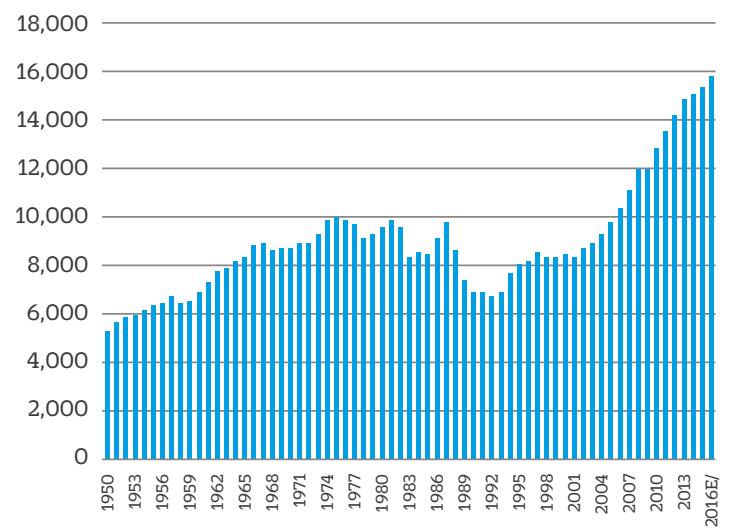

Fuente: BCRP e INEI.

Elaboración propia.
Asimismo, del gráfico 3 se puede destacar la participación importante del sector de servicios, que deja como eufemismos las afirmaciones que señalan al Perú como país agrícola, pesquero o minero, y que cobran sentido cuando se revisa la composición de las exportaciones mostrada en el gráfico 4A.

Gráfico 3. Perú: PBI por sectores económicos, 19502016 (en millones de soles de 2007)

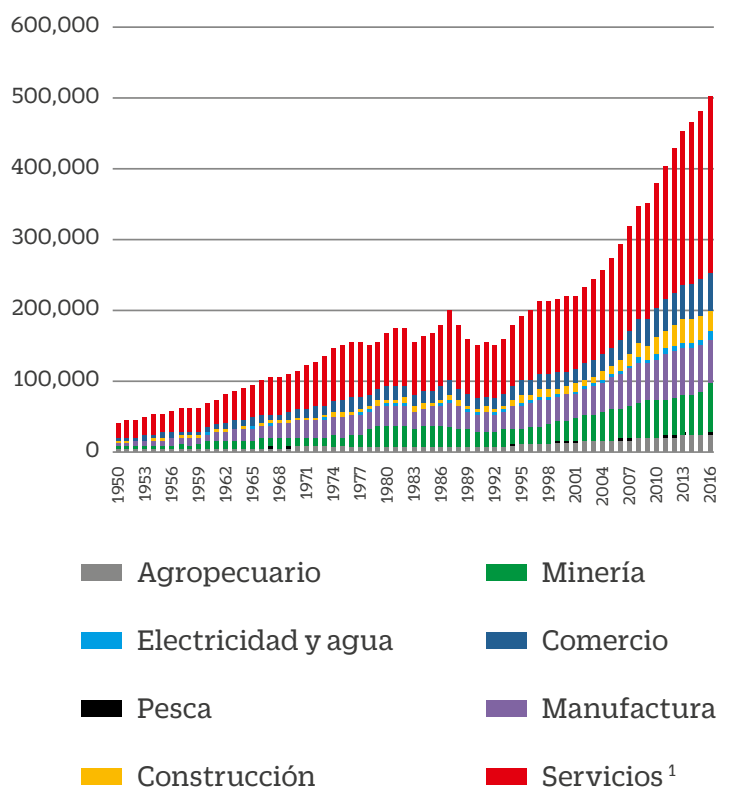

1/ Incluye derechos de importación e impuestos a los productos. Fuente: BCRP e INEI.

Elaboración propia

\section{Gráfico 4A. Perú: Exportaciones totales por tipo de producto, 1950-2016 (en millones de dólares estadounidenses)}

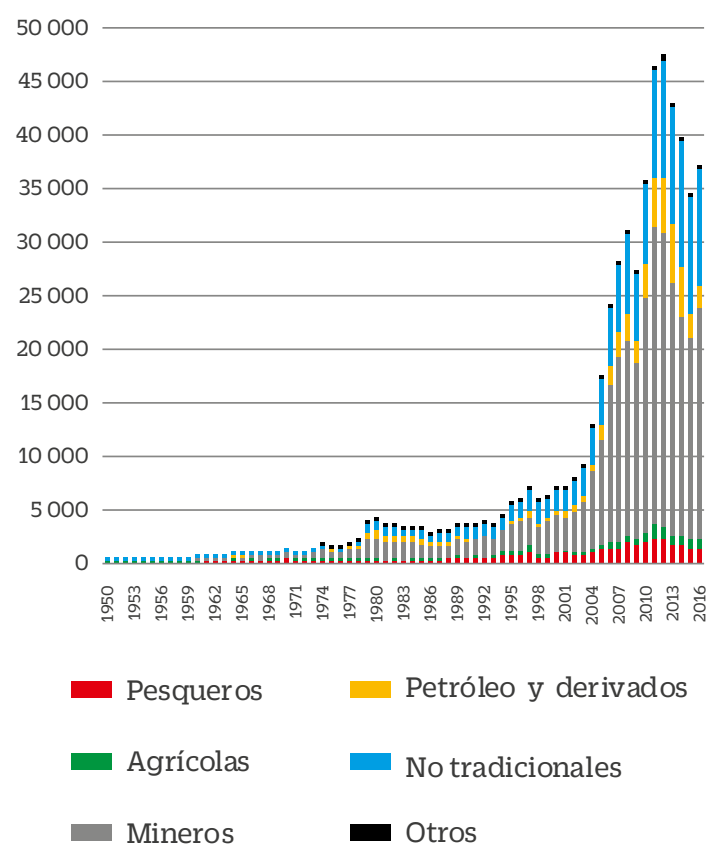

Fuente: BCRP

Elaboración propia. 
Resulta más realista la afirmación de que el Perú ha sido, y sigue siendo, un país primario exportador, no obstante los grandes avances conseguidos en la diversificación de los productos que ofrece en los mercados internacionales (ver gráfico 4B).

Gráfico 4B. Perú: Exportaciones totales por tipo de producto, 1950-1978 (en millones de dólares estadounidenses)

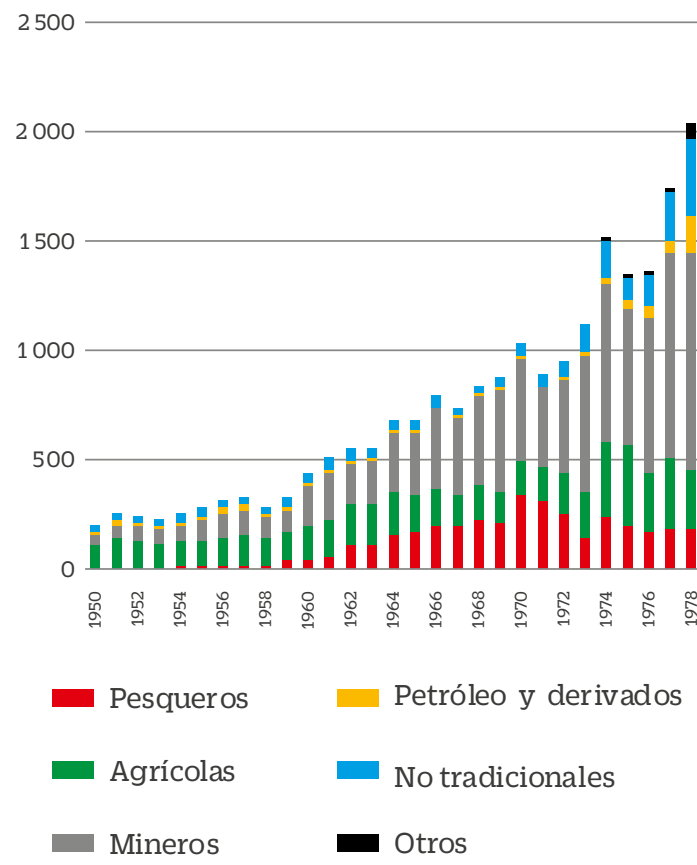

Fuente: BCRP

Elaboración propia

Una revisión de la evolución del PBI, en general, y de las exportaciones totales, en particular (ver gráfico 5), da cuenta de un notorio punto de quiebre a partir de la década de 1990, que indicaría una correlación entre estas variables. Cabe recordar que los principales hechos ocurridos en dicho momento fueron la reinserción del Perú en la economía mundial (ver gráfico 6) y el paquete de reformas para reactivar la economía (ver gráfico 7), que permitieron acabar con la hiperinflación y encaminar de vuelta la actividad económica local de forma sostenida.
Gráfico 5. Perú: Exportaciones y PBI total, 1950-2016 (en millones de soles de 2007)

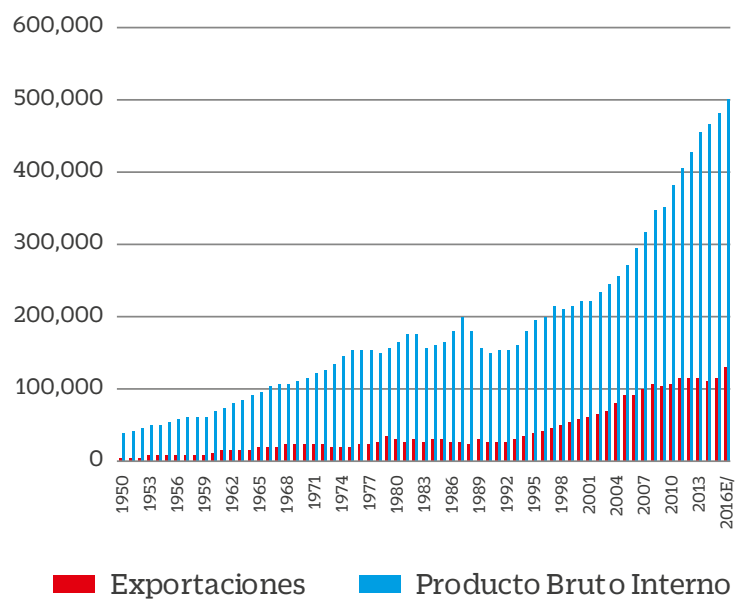

Fuente: INEI

Elaboración propia

Gráfico 6. Perú: Grado de apertura comercial, 1950 2016 (en porcentaje)

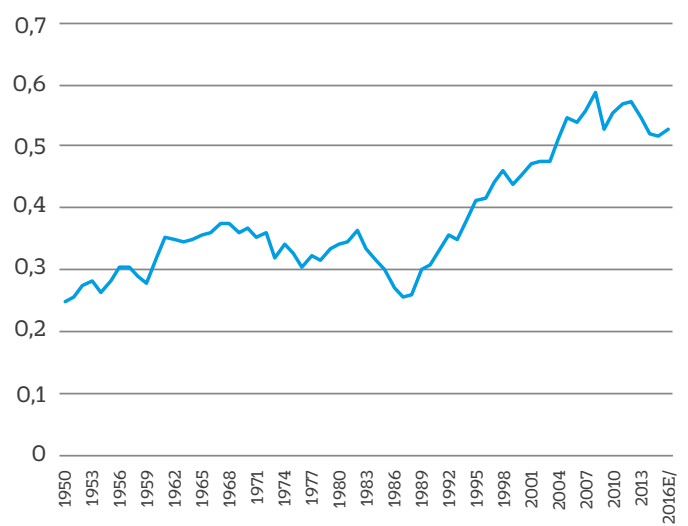

Fuente: INEI

Elaboración propia.

Gráfico 7. Perú: Tasa de crecimiento anual del PBI total y de las exportaciones totales, 1951-2016 (en porcentaje)

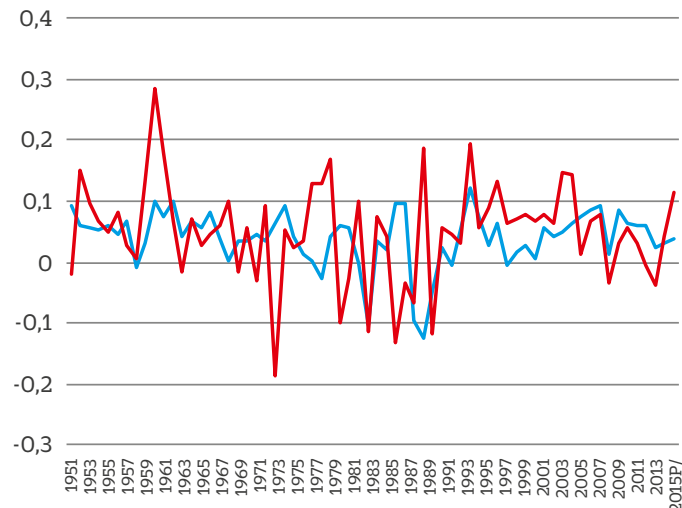

PBI total

Exportaciones

Euente: INEI

Elaboración propia 
En línea con lo anterior, si se analiza el comportamiento de las exportaciones totales (ver gráfico 8), se notará claramente el impacto de las acciones para la reinserción del Perú en la economía mundial, combinadas con las ayudas unilaterales recibidas por parte de Estados Unidos², las ampliaciones de los sistemas generalizados de preferencias (SGP) de Japón y de la Unión Europea, y la entrada en vigencia de los tratados de libre comercio (TLC) ${ }^{3}$.

Gráfico 8. Perú: Exportaciones totales, 1950-2016 (en millones de soles de 2007)

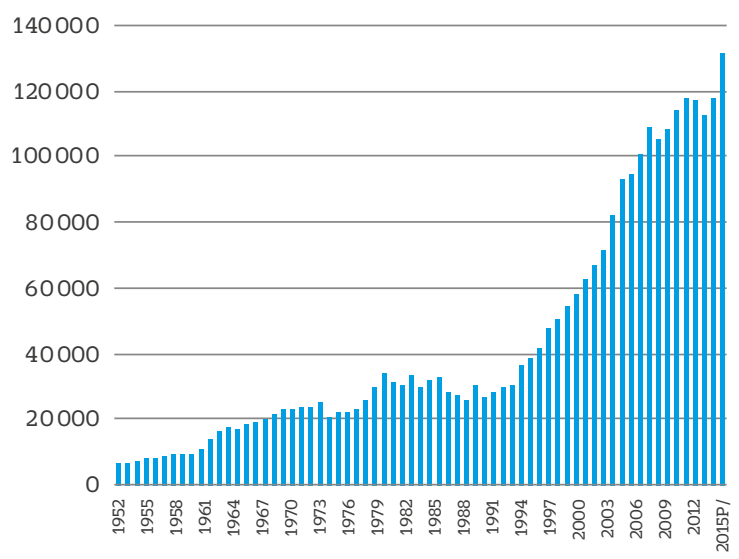

Fuente: INEI

Elaboración propia

Hasta se podría afirmar que la dinámica ocurrida en el sector externo es lo que ha empujado al desempeño mostrado por el PBI, luego de revisar las cifras sobre la participación de las exportaciones en el PBI que se presentan a continuación:

\section{Gráfico 9. Perú: Participación de las exportaciones totales en el PBI total, 1950-2016 (en porcentaje)}

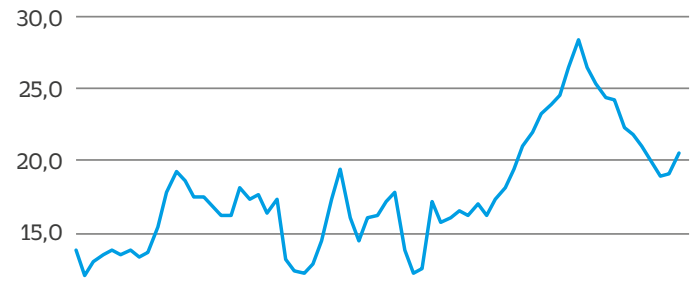

10,0

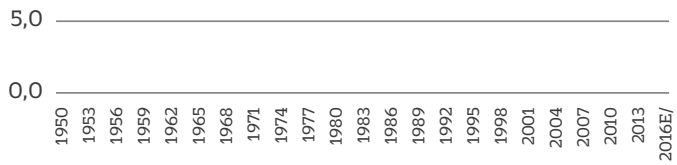

Fuente: INEI

Elaboración propia.

2. La Ley de Preferencias Arancelarias Andinas (ATPA) y la Ley para la Erradicación de Drogas y Promoción del Comercio Andino (ATPDEA)

3. El primer TLC en entrar en vigencia fue el Acuerdo de Promoción Comercial (APC) Perú-Estados Unidos, del 1 de febrero de 2009 .
Mención especial merecen las exportaciones mineras, que han llegado a representar la quinta parte del PBI, como se puede ver en el gráfico 10.

Gráfico 10. Perú: Participación de las exportaciones mineras en el PBI total, 1950-2016 (en porcentaje)

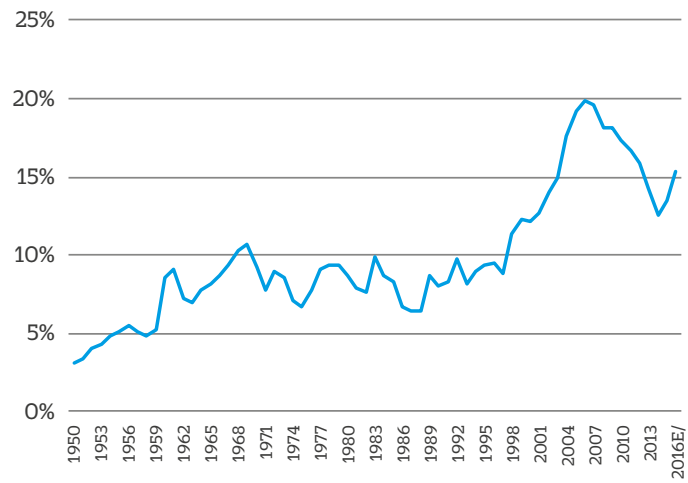

Fuente: BCRP e INEI

Elaboración propia.

Una revisión más exhaustiva de las exportaciones peruanas desde 1950 hasta la actualidad deja ver la gran importancia de la minería, que deja atrás al sector agrícola —el cual tuvo su periodo de gloria en la década de 1950o al pesquero - que lo tuvo a inicios de la década de 1970 - tal como se aprecia en el gráfico 11.

Gráfico 11. Perú: Participación de las exportaciones tradicionales en el total exportado, 1950-2016 (en porcentaje)

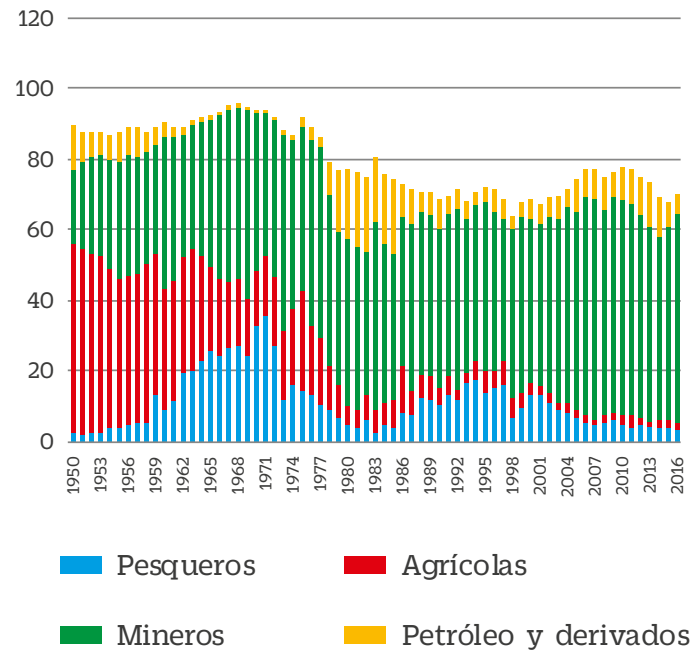

Fuente: BCRP

Elaboración propia

Más específicamente, las exportaciones mineras tienen una participación que, en promedio, ha aumentado (a inicios de la década de 1950 fue de 20\%), llegando a sobrepasar de manera continua al 50\% desde 2003 (ver gráfico 12A). 


\section{Gráfico 12A. Perú: participación de las exportaciones mineras en el total exportado, 1950-2016 (en porcentaje)}

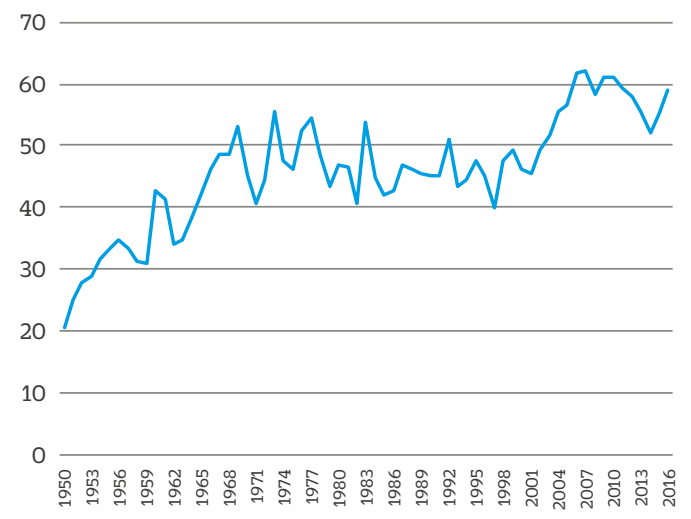

Fuente: BCRP.

Elaboración propia.

Entre los productos mineros de exportación, han destacado el cobre, el oro, la plata, el zinc, el hierro, el estaño y el plomo, entre otros. En los últimos diez años lo vienen haciendo el cobre, el oro, el plomo y el zinc, tal como se aprecia en el gráfico 12B, por lo cual nos concentraremos en el análisis de los precios internacionales, los volúmenes exportados y el valor exportado de estos metales, para aproximar un pronóstico de la tendencia de las exportaciones totales. Ello se desarrollará en las siguientes secciones.

\section{Gráfico 12B. Perú: estructura de las exportaciones mineras, enero 2009-setiembre 2017 (en millones de dólares)}

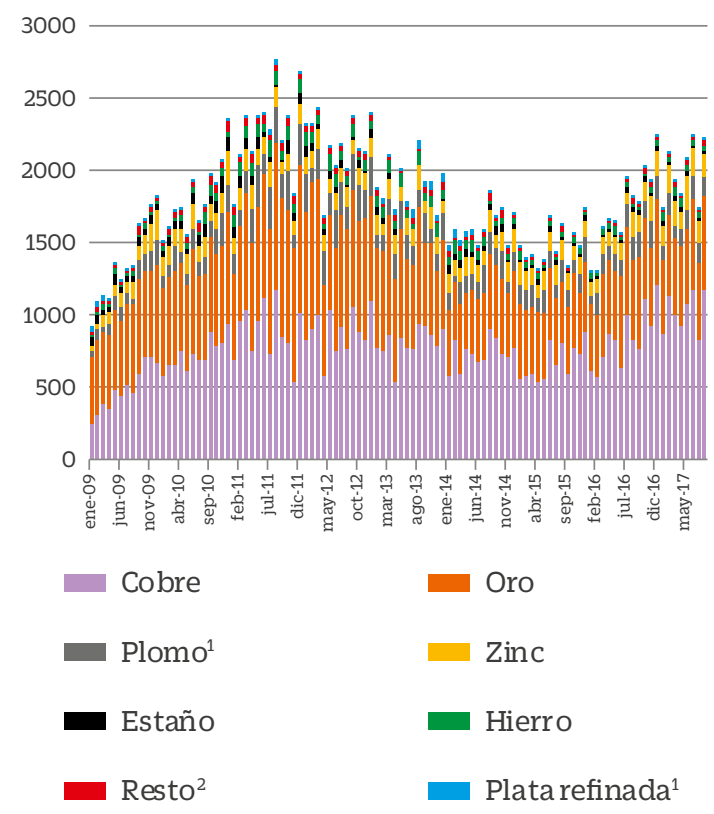

1/ Incluye contenidos de plata.

2/ Incluye molibdeno, bismuto y tungsteno,

principalmente.

Fuente: BCRP.

Elaboración propia.

\section{Metodología para la Proyección de Exportaciones de los Principales Metales de Exportación}

En una primera etapa, se evaluará el nivel de volatilidad de los precios internacionales de los cuatro metales con mayor participación en las exportaciones, a partir del análisis de los estadísticos descriptivos tradicionales; en particular, el coeficiente de variación a través de la siguiente expresión:

$$
C V\left(X_{j}\right)=\left[\frac{\operatorname{Media}\left(X_{j}\right)}{S D\left(X_{j}\right)}\right] \times 100
$$

Los resultados de la volatilidad fundamentarán la capacidad de pronóstico de las respectivas exportaciones, sobre la base de un escenario optimista.

Luego de ello, se efectuará un análisis de las características aleatorias y con dirección de las variables, a fin de encontrar una representación funcional de la serie de tiempo asociada a sus propios valores desfasados y a sus aspectos estocásticos (probabilísticos) presentes y pasados. Ello pasa por aplicar las pruebas de raíz unitaria aleatoria en niveles, para verificar si corresponde o no integrarlas en sus primeras diferencias para volverlas estacionarias.

Para esto, se trabaja una secuencia simple para el test aumentado de raíces unitarias de Dickey-Fuller (1979), en virtud del siguiente procedimiento:

Sea $X_{t}$ una serie económica de tiempo, como cada una de las sujetas de análisis. Entonces, partiendo de

(1) $X_{t}=\delta+\rho X_{t-1}+\varepsilon_{t}$

donde $\delta$ es el punto de interceptación de la tendencia, $\rho$ es el grado de relación entre momentos sucesivos de la serie y $\varepsilon$ es el error aleatorio.

Si es posible demostrar que $\rho$ tiende a 1 para cualquiera de las variables consideradas, se tendrá entonces que

(2) $X_{t}=\delta+X_{t-1}+\varepsilon_{t}$

de donde, acomodando términos, se tiene

(3) $X_{t}-X_{t-1}=\delta+\varepsilon_{t}$

Y, por ende,

(4) $\Delta X_{t}=\delta+\varepsilon_{t}$

por lo que la variación de la variable de un periodo a otro $\left(\Delta X_{t}\right)$ tendrá una dirección para su tendencia, la cual valuará un punto de corte en el eje de las ordenadas, pero no dejará de ser aleatoria. Las series de tiempo con estas características exhiben en su comportamiento una suerte de "recorrido aleatorio" (i. e., random walk) con dirección, no estacionario, por lo que poseen raíces unitarias aleatorias y no se encuentran integrados, al menos cuando son considerados a niveles (i. e., sin diferencias).

Entonces, haciendo extensiva esta idea abarcando tres rezagos, para de esta manera considerar subperiodos trimestrales, se tiene

(5) $\Delta X_{t}=\delta+\gamma X_{t-1}+\beta_{1} \Delta X_{t-1}+\beta_{2} \Delta X_{t-2}+\beta_{3} \Delta X_{t-3}+\varepsilon_{t}$ 
Desarrollando el lado endógeno, se tiene

(6) $X_{t}-X_{t-1}=\delta+\gamma X_{t-1}+\beta_{1} \Delta X_{t-1}+\beta_{2} \Delta X_{t-2}+\beta_{3} \Delta X_{t-3}+\varepsilon_{t}$ $Y$, despejando, se llega a

(7) $X_{t}=\delta+(1+\gamma) X_{t-1}+\beta_{1} \Delta X_{t-1}+\beta_{2} \Delta X_{t-2}+\beta_{3} \Delta X_{t-3}+\varepsilon_{t}$ Nótese que la ecuación (7) es igual a la ecuación (4), con la salvedad de la consideración de los rezagos endógenos, por lo que, si esta ecuación es estimada y no es posible rechazar la hipótesis de que $\gamma-0$, entonces, indirectamente, se estaría demostrando que $\rho-0$, lo que implicaría la no estacionariedad de la variable a niveles. En ese supuesto, puede adaptarse el procedimiento para aplicárselo a la primera diferencia de la variable, como se muestra a continuación:

(8) $\Delta X_{t}=\delta+\rho \Delta X_{t-1}+\varepsilon_{t}$

Por lo que, si se demuestra que $\rho$ tiende a 1 , se tendrá entonces que

(9) $\Delta X_{t}=\delta+\Delta X_{t-1}+\varepsilon_{t}$

De donde, arreglando términos, se tiene que

(10) $\Delta X_{t}-\Delta X_{t-1}=\delta+\varepsilon_{t}$

$Y$, por ende,

(11) $\Delta^{2} X_{t}=\delta+\varepsilon_{t}$

Por lo que la variación de la variable (i.e., $\Delta X_{t}$ ) de un periodo a otro (i.e., $\Delta^{2} X_{t}$ ) tendrá una dirección para su tendencia, la cual implicará un punto de corte en el eje de las ordenadas, pero no dejará de ser aleatoria. En caso de que ocurra lo contrario, puede hacerse extensiva esta idea abarcando tres rezagos para considerar subperiodos trimestrales, así:

(12) $\Delta^{2} X_{t}=\delta+\gamma \Delta X_{t-1}+\beta_{1} \Delta^{2} X_{t-1}+\beta_{2} \Delta^{2} X_{t-2}+\beta_{3} \Delta^{2} X_{t-3}+\varepsilon_{t}$ Desarrollando el lado endógeno, se tiene

(13) $\Delta \Delta X_{t}=\delta+\gamma \Delta X_{t-1}+\beta_{1} \Delta^{2} X_{t-1}+\beta_{2} \Delta^{2} X_{t-2}+\beta_{3} \Delta^{2} X_{t-3}+\varepsilon_{t}$

(14) $\Delta\left(X_{t}-X_{t-1}\right)=\delta+\gamma \Delta X_{t-1}+\beta_{1} \Delta^{2} X_{t-1}+\beta_{2} \Delta^{2} X_{t-2}+\beta_{3} \Delta^{2} X_{t-3}+\varepsilon_{t}$

(15) $\Delta X_{t}-\Delta X_{t-1}=\delta+\gamma \Delta X_{t-1}+\beta_{1} \Delta^{2} X_{t-1}+\beta_{2} \Delta^{2} X_{t-2}+\beta_{3} \Delta^{2} X_{t-3}+\varepsilon_{t}$

(16) $\Delta X_{t}=\delta+(1+\gamma) \Delta X_{t-1}+\beta_{1} \Delta^{2} X_{t-1}+\beta_{2} \Delta^{2} X_{t-2}+\beta_{3} \Delta^{2} X_{t-3}+\varepsilon_{t}$

La ecuación (16) representa lo mismo que la ecuación (11), con la salvedad de la consideración de los rezagos, por lo que, si esta ecuación es estimada y no es posible rechazar la hipótesis de que $\gamma-1$, entonces indirectamente se estaría demostrando que $\rho-1$, lo que implica la no estacionariedad de la variable también a su primera diferencia

Una vez que se identifiquen las características de las series, se procederá a realizar los pronósticos considerando escenarios, para lo que se requiere identificar la curva de tendencia que sea compatible con el recorrido asociado al comportamiento observado de los datos.

\section{APLICACIÓN Y RESULTADOS}

En esta sección, contemplamos las siguientes variables para construir nuestro modelo:

\begin{tabular}{|c|l|}
\hline Clave & \multicolumn{1}{|c|}{ Variable } \\
\hline XCOB & Exportaciones peruanas de cobre en millones de dólares \\
\hline XCOBV & $\begin{array}{l}\text { Volumen de las exportaciones peruanas de cobre en miles } \\
\text { de toneladas }\end{array}$ \\
\hline XORO & Exportaciones peruanas de oro en millones de dólares \\
\hline XOROV & $\begin{array}{l}\text { Volumen de las exportaciones peruanas de oro en miles de } \\
\text { onzas troy }\end{array}$ \\
\hline XPLO & Exportaciones peruanas de plomo en millones de dólares \\
\hline XPLOV & $\begin{array}{l}\text { Volumen de las exportaciones peruanas de plomo en miles } \\
\text { de toneladas }\end{array}$ \\
\hline XZNC & Exportaciones peruanas de zinc en millones de dólares \\
\hline XZNCV & $\begin{array}{l}\text { Volumen de las exportaciones peruanas de zinc en miles de } \\
\text { toneladas }\end{array}$ \\
\hline PCOB & Precio internacional del cobre en centavos de dólar por libra \\
\hline PORO & Precio internacional del oro en dólares por onza troy \\
\hline PPLO & Precio internacional del plomo en centavos de dólar por libra \\
\hline PZNC & Precio internacional del zinc en centavos de dólar por libra \\
\hline TCBP & $\begin{array}{l}\text { Tipo de cambio bancario promedio en soles por dólar } \\
\text { estadounidense }\end{array}$ \\
\hline
\end{tabular}

Los datos de estas variables están en periodicidad mensual, con una muestra que va desde enero de 2009 hasta setiembre de 2017. Las fuentes de los datos son el Banco Central de Reserva del Perú (BCRP) y la Superintendencia Nacional de Administración Tributaria (SUNAT). Todas las cifras consideradas han sido obtenidas a partir de la página web del BCRP, en su sección de estadísticas económicas.

Los gráficos 13, 14, 15 y 16 presentan, en escala dual, la variación conjunta del valor de las exportaciones de cada mineral considerado con su respectivo precio en los mercados internacionales. Al tratarse de exportaciones mineras tomadas en cuenta con sus respectivos valores en dólares, era de esperarse una fuerte relación con sus cotizaciones en los mercados internacionales, como parece apreciarse en los gráficos mencionados. Sin embargo, esto ocurre en el caso de las exportaciones de oro, plomo y zinc; no así en las de cobre. Al respecto, observando la matriz de correlación simple presentada en la tabla 1, se encuentra que se ha dado una correlación baja de 29,99\%, siendo en cambio bastante mayor y con signo negativo la correlación encontrada con el volumen de dichas exportaciones $(-53,45 \%)$, resultado compatible con un escenario de crecimiento para las exportaciones cupríferas en el marco de fuertes fluctuaciones de su precio en los mercados internacionales. 
Gráfico 13. Valor de las exportaciones de Precio Internacional del Cobre

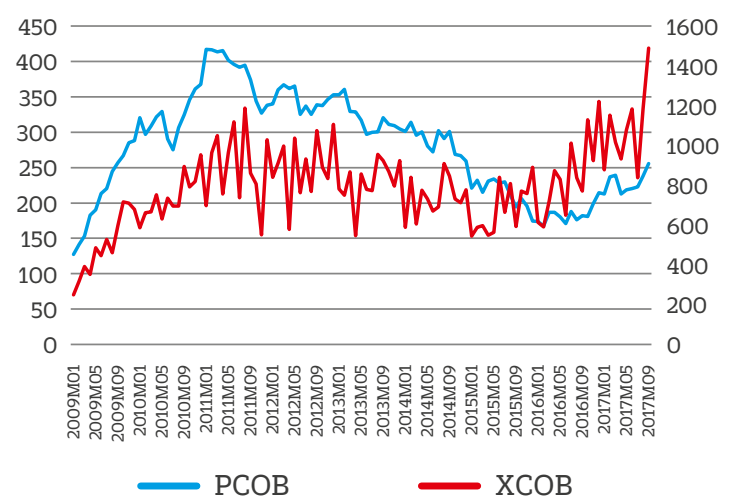

Fuente: Elaboración propia.

Gráfico 14. Valor de las exportaciones de Precio Internacional del Oro
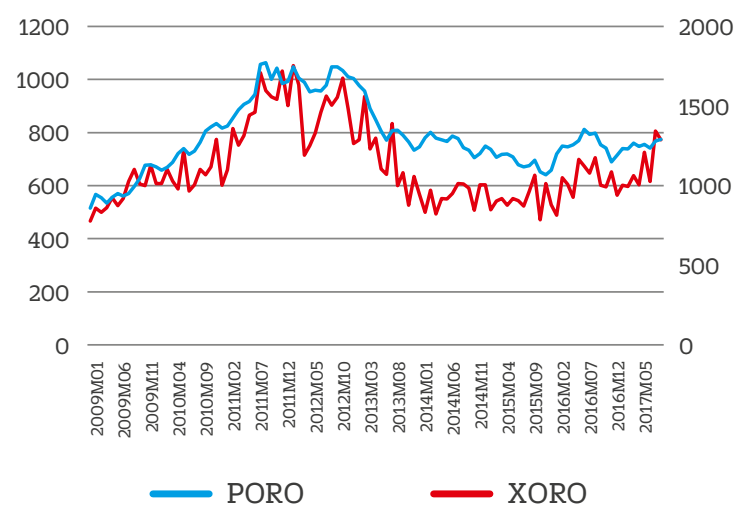

Fuente: Elaboración propia.

Gráfico 15. Valor de las exportaciones de Precio Internacional del Plomo

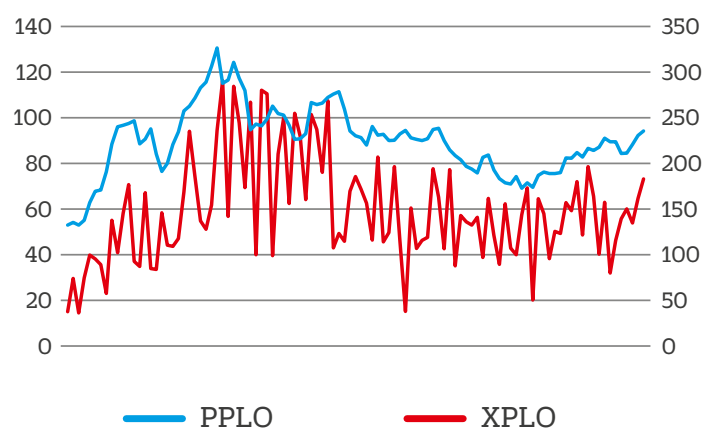

Fuente: Elaboración propia.
Gráfico 16. Valor de las exportaciones de Precio Internacional del Zinc

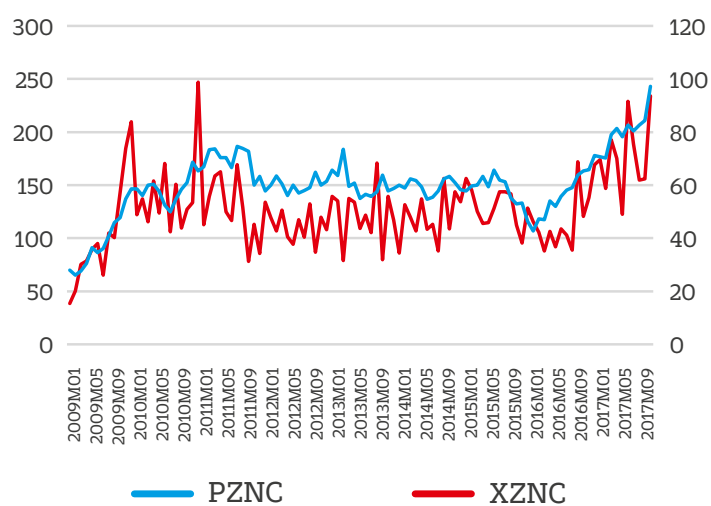

Fuente: Elaboración propia.

Tabla 1. Matriz de correlación simple

\begin{tabular}{|c|c|c|c|}
\hline & $\mathrm{XCOBV}$ & $\mathrm{XCOB}$ & PCOB \\
\hline XCOBV & 1,000000 & 0,620815 & $-0,534565$ \\
\hline ХCOB & 0,620815 & 1,000000 & 0,299965 \\
\hline \multirow[t]{2}{*}{ РСOB } & $-0,534565$ & 0,299965 & 1,000000 \\
\hline & XOROV & XORO & PORO \\
\hline XOROV & 1,000000 & 0,538078 & 0,022212 \\
\hline XORO & 0,538078 & 1,000000 & 0,851172 \\
\hline \multirow[t]{2}{*}{ PORO } & 0,022212 & 0,851172 & 1,000000 \\
\hline & XPLOV & XPLO & PPLO \\
\hline XPLOV & 1,000000 & 0,908851 & 0,174766 \\
\hline XPLO & 0,908851 & 1,000000 & 0,545963 \\
\hline \multirow[t]{2}{*}{ PPLO } & 0,174766 & 0,545963 & 1,000000 \\
\hline & XZNCV & XZNC & PZNC \\
\hline XZNCV & 1,000000 & 0,678535 & $-0,158874$ \\
\hline XZNC & 0,678535 & 1,000000 & 0,601386 \\
\hline PZNC & $-0,158874$ & 0,601386 & 1,000000 \\
\hline
\end{tabular}

Elaboración propia.

Las exportaciones de cobre son un rubro importante de la oferta exportadora global del Perú, pues representan aproximadamente un cuarto del total, por lo que todo lo que ocurra con las mismas, así como en la evolución de su precio internacional, afectará de manera significativa el total de exportaciones, y, por ende, la balanza comercial de la nación. Dicha variable experimentó una tendencia a la baja en buena parte del periodo considerado, mostrando su valor más bajo en enero de 2015, con 544,7930 millones de dólares. A partir de esa fecha, la variable se mantuvo unos meses con pequeñas fluctuaciones, manifestando una tendencia estable para luego iniciar una lenta recuperación, 
la cual conjuga con las expectativas actuales de mayor crecimiento. Este comportamiento puede apreciarse en el gráfico 13.

Similar panorama se muestra en el caso del valor de las exportaciones de oro, plomo y zinc, que le siguen en importancia relativa, tal como se evidencia al observar los gráficos 14, 15 y 16

Seguidamente, de acuerdo con los resultados de la tabla 2 de estadísticos descriptivos, y con el objetivo de evaluar el nivel de volatilidad de los precios internacionales del valor de las exportaciones, se obtienen los coeficientes de variación de las mismas.

\section{Tabla 2. Estadísticos descriptivos de los precios internacionales}

\begin{tabular}{|l|r|r|r|r|}
\hline & PCOB & \multicolumn{1}{|c|}{ PORO } & \multicolumn{1}{|c|}{ PPLO } & \multicolumn{1}{|c|}{ PZNC } \\
\hline Mean & 279,5224 & 1309,889 & 89,49146 & 59,44760 \\
\hline Median & 293,3854 & 1262,915 & 90,02354 & 59,79814 \\
\hline Maximum & 417,1232 & 1772,706 & 130,2121 & 84,30195 \\
\hline Minimum & 127,5755 & 860,1476 & 52,56825 & 26,01726 \\
\hline Std. Dev. & 72,15567 & 223,5175 & 15,22330 & 11,37385 \\
\hline Skewness & $-0,040456$ & 0,404635 & 0,004448 & $-0,589889$ \\
\hline Kurtosis & 2,032168 & 2,558848 & 3,266827 & 4,256946 \\
\hline Jarque-Bera & 4,087394 & 3,681308 & 0,308861 & 12,87774 \\
\hline Probability & 0,129549 & 0,158714 & 0,856903 & 0,001598 \\
\hline Sum & 29070,33 & 136228,4 & 9307,111 & 6182,550 \\
\hline Sum Sq. Dev. & 536263,3 & 5145889 & 23870,13 & 13324,53 \\
\hline Observations & 104 & 104 & 104 & 104 \\
\hline Coef. de variación & $25,81391 \%$ & $17,06385 \%$ & $17,01279 \%$ & $19,13249 \%$ \\
\hline
\end{tabular}

Elaboración propia.

El precio del cobre ha sido claramente la variable de mayor fluctuación en el periodo en estudio. A ella le siguen los precios del zinc, el oro y el plomo, aunque con muy poca diferencia. Estos resultados fundamentarán la capacidad de pronóstico de las exportaciones de dichos minerales, basándose en un escenario optimista.

Al analizar las características aleatorias y con dirección de las variables, tanto a niveles como a su primera diferencia, se encuentra que, salvo en el caso de los volúmenes de exportación del plomo y el zinc (XPLOV Y XZNCV, respectivamente), y del precio internacional del plomo (PPLO), que resultaron integradas a niveles, el resto de variables se hicieron estacionarias a su primera diferencia. La tabla 3 presenta un resumen del resultado del test de Dickey-Fuller a todas las variables.
Tabla 3. Resumen del test aumentado de DickeyFuller sobre raíces unitarias

\begin{tabular}{|l|c|c|c|}
\hline VARIABLE & $\mathrm{d}=0$ & $\mathrm{~d}=1$ & RESULTADO \\
\hline XCOB & $-2,124581$ & $-6,136643$ & Integrada $-\mathrm{I}(\mathrm{d}=1)$ \\
\hline XORO & $-1,071131$ & $-5,849638$ & Integrada $-\mathrm{I}(\mathrm{d}=1)$ \\
\hline XPLO & $-1,757231$ & $-5,500000$ & Integrada $-\mathrm{I}(\mathrm{d}=1)$ \\
\hline XZNC & $-2,627668$ & $-6,882400$ & Integrada $-\mathrm{I}(\mathrm{d}=1)$ \\
\hline & & & \\
\hline & & & \\
\hline XCOBV & $-1,603005$ & $-7,098231$ & Integrada $-\mathrm{I}(\mathrm{d}=1)$ \\
\hline XOROV & $-2,203842$ & $-6,993815$ & Integrada $-\mathrm{I}(\mathrm{d}=1)$ \\
\hline XPLOV & $-3,635887$ & $-8,175322$ & Integrada $-\mathrm{I}(\mathrm{d}=0)$ \\
\hline XZNCV & $-3,570880$ & $-8,087105$ & Integrada - $\mathrm{I}(\mathrm{d}=0)$ \\
\hline & & & \\
\hline PCOB & $-3,008053$ & $-4,656802$ & Integrada $-\mathrm{I}(\mathrm{d}=1)$ \\
\hline PORO & $-2,231802$ & $-5,000004$ & Integrada $-\mathrm{I}(\mathrm{d}=1)$ \\
\hline PPLO & $-3,584788$ & $-4,856964$ & Integrada $-\mathrm{I}(\mathrm{d}=0)$ \\
\hline PZNC & $-1,860451$ & $-4,631945$ & Integrada $-\mathrm{I}(\mathrm{d}=1)$ \\
\hline
\end{tabular}

Elaboración propia.

La selección de los rezagos endógenos para la prueba de Dickey-Fuller se ha hecho, en parte, de acuerdo con el objetivo de trabajar trimestres (tres rezagos), sustentando la decisión con los estadísticos de criterios de error ${ }^{4}$. Cabe recordar que los resultados del estadístico en esta prueba son evaluados con los valores críticos de MacKinnon (1996), resultados que también se evidencian aplicando la prueba de Phillips-Perron (1988).

Tomando en cuenta las características seriales mencionadas, se procede a hacer pronósticos para el valor de las exportaciones peruanas de cobre, oro, plomo y zinc, considerando un escenario entre optimista y moderadamente optimista. La razón para ello se fundamenta en el comportamiento observado para estas variables en el periodo contemplado y, sobre todo, en las expectativas de los agentes económicos.

En el periodo analizado se observan caídas en los precios internacionales de estos minerales, especialmente en el caso del cobre, principal ítem de la oferta exportadora peruana, llegando a niveles mínimos, pero a partir de los cuales se ha producido una recuperación importante en los mismos, y, como resultado de ello, una consecuente subida en los valores exportados. Este recorrido es compatible con una curva de tendencia cuadrática, cuyos valores medios en las ordenadas no se distancian mayormente en un 5\% de sus extremos volátiles, representada por esta ecuación:

(17) $X_{t}=a+b T_{t}+c T_{t}^{2}+\varepsilon_{t}$

Siendo $X_{t}$ cualquiera de las variables consideradas y $T$ el vector considerador del tiempo en periodicidad mensual, mientras que $c T$ sigue siendo el vector considerador 
de los errores, que, en este caso, está representando la eventualidad de las fluctuaciones observadas.

Esta misma tendencia es empleada en los pronósticos asumiendo continuidad en la recuperación y una fase expansiva del ciclo económico, lo cual es compatible con las expectativas de los agentes económicos y las del Banco Mundial (2017). Algunos factores restrictivos infaltables podrían originar fuerzas en sentido contrario, con relación a eventuales conflictos internos del sector minero, huelgas salariales o medidas políticas que limiten la extracción minera; y, por el lado externo, la posible atenuación del crecimiento económico de China, nuestro principal socio comercial. En todo caso, estos factores están contemplados en el área de dispersión demarcada por las bandas + $1-2$ de desviación estándar, compatible con un intervalo de confianza de $95 \%$.

Las secuencias de las proyecciones se aprecian en los gráficos del 17 al 20.

\section{Gráfico 17. Valor de las exportaciones de Cobre: Pronóstico a 2021}

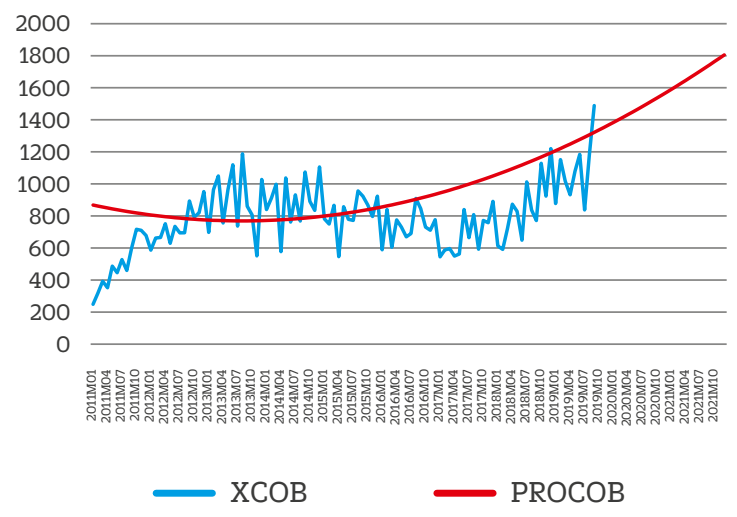

Fuente: Elaboración propia.

Gráfico 18. Valor de las exportaciones de Oro: Pronóstico a 2021

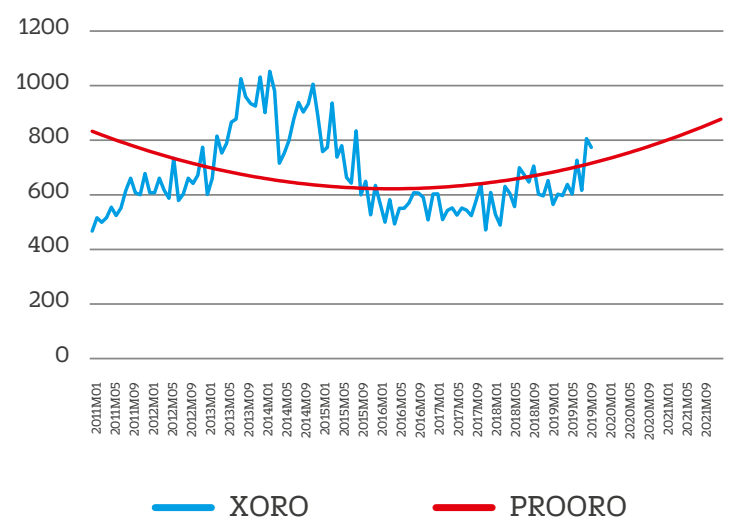

Fuente: Elaboración propia.
Gráfico 19. Valor de las exportaciones de Plomo: Pronóstico a 2021

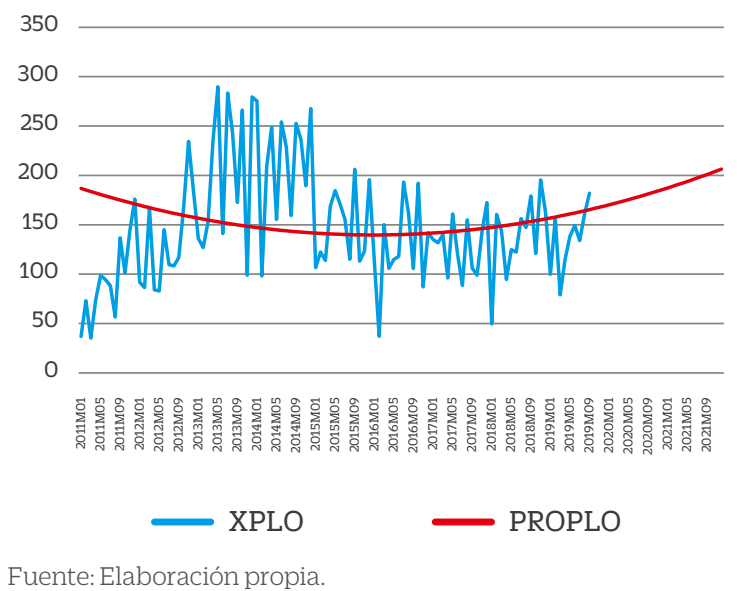

Gráfico 20. Valor de las exportaciones de Plomo: Pronóstico a 2021

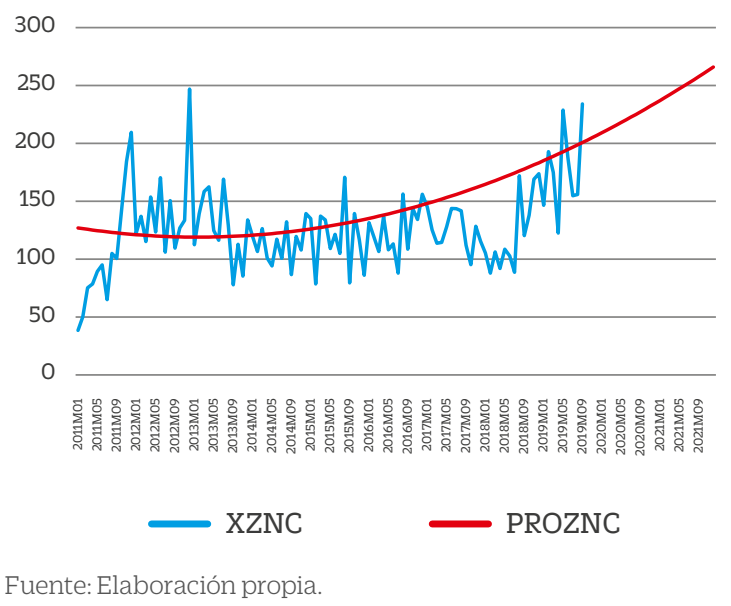

Los valores proyectados para 2021 se presentan en la tabla 4: 
Tabla 4. Pronóstico de las exportaciones para 2021 (en millones de dólares)

\begin{tabular}{|l|r|r|r|r|}
\hline Obs. & PROCOB & PROORO & PROPLO & PROZNC \\
\hline 2021M01 & 1590,016 & 801,5505 & 187,3073 & 237,1353 \\
\hline 2021M02 & 1608,551 & 807,8345 & 188,9033 & 239,6260 \\
\hline $2021 M 03$ & 1627,294 & 814,2266 & 190,5256 & 242,1428 \\
\hline $2021 M 04$ & 1646,243 & 820,7269 & 192,1742 & 244,6856 \\
\hline 2021M05 & 1665,400 & 827,3353 & 193,8492 & 247,2544 \\
\hline 2021M06 & 1684,763 & 834,0519 & 195,5504 & 249,8493 \\
\hline 2021M07 & 1704,333 & 840,8766 & 197,2779 & 252,4701 \\
\hline 2021M08 & 1724,111 & 847,8095 & 199,0318 & 255,1169 \\
\hline 2021M09 & 1744,095 & 854,8505 & 200,8119 & 257,7898 \\
\hline 2021M10 & 1764,286 & 861,9996 & 202,6184 & 260,4886 \\
\hline 2021M11 & 1784,684 & 869,2569 & 204,4512 & 263,2135 \\
\hline 2021M12 & 1805,290 & 876,6223 & 206,3103 & 265,9643 \\
\hline PROCOB & Pronóstico de las exportaciones de cobre. Millones de dólares. \\
\hline PROORO & Pronóstico de las exportaciones de oro. Millones de dólares. \\
\hline PROPLO & Pronóstico de las exportaciones de plomo. Millones de dólares. \\
\hline PROZNC & Pronóstico de las exportaciones de zinc. Millones de dólares \\
\hline
\end{tabular}

Fuente: Elaboración propia.

Los diversos equipos económicos que han estado al frente de la economía peruana siempre han tenido como meta la mayor diversificación de la oferta exportadora. Los resultados están a la vista, con el importante incremento experimentado en las exportaciones no tradicionales en diversos rubros, explotando de esta manera las grandes oportunidades aparecidas como consecuencia de los TLC suscritos por el Perú. Sin embargo, este crecimiento no es aún significativo como para que perdamos etiquetas históricas, como aquellas que clasifican al Perú como un país minero, uno exportador de materias primas o uno dependiente del vaivén de los precios internacionales, panorama que no cambiará, al menos, para el bicentenario de la independencia política.

\section{CONCLUSIONES Y RECOMENDACIONES}

La evidencia presentada en este artículo ha servido para reflexionar sobre que la condición que tiene el Perú de ser un país primario exportador, en particular exportador de minerales, no es mala, así como para evidenciar los grandes avances en la diversificación de exportaciones, reflejados en el desempeño de las exportaciones no tradicionales.

Vista la dependencia en la evolución de los precios internacionales de los metales es que se hicieron los pronósticos, que auguran tendencias crecientes, lo cual, unido a mayores volúmenes exportados, ante los requerimientos que se esperan por parte de China, permitiría seguir en la senda creciente del valor exportado.
En este contexto, se recomienda continuar con la tarea de diversificar las exportaciones de bienes y servicios. Para ello, se requiere mejorar la infraestructura tanto física como virtual, y revisar aquellos factores que están restándoles competitividad a los bienes y a los servicios exportados. Además, se precisa hacer un buen uso de los recursos generados por el canon minero, a fin de que una mayor parte de la población pueda acceder a la bonanza económica, de modo que, con ello, vea mejorada su calidad de vida.

\section{REFERENCIAS}

Akaike, H. (1984). A New Look at the Statistical Identification Model. IEEE Transactions on Automatic Control, 19(6), 716-723.

Bhagwati, J. N., Panagariya, A. \& Srinivasan, T. N. (1998). Lectures on International Trade. Cambridge: The MIT Press.

Braun, M., Feldman, G., Junowicz, M. \& Roitman, A. (2008). El desarrollo de nuevos sectores de exportación en América Latina: lecciones de 10 casos exitosos. Avance de Investigación 15. Madrid: Fundación Carolina.

Deardorff, A. (1984). Testing Trade Theories and Predicting Trade Flows. En R. Jones \& P. Kenem (eds), Handbook of International Economics. Volumen I. Ámsterdam: North-Holland.

Dickey, D. \& Fuller, W. (1979). Distribution of the estimators for autoregressive time series with a unit root. Journal of American Statistics Association, 74(366), 426-431.

Diebold, F. (1977). Elements of Forecasting. Cincinnati, Ohio: Southwestern College Publishing.

Engel, R. F. \& Granger, C. W. J. (1987). Co-integration and error correction: Representation, estimation, and testing. Econometrica, 55(2), 251-276

Feenstra, R. (1998). Integration of trade and disintegration of production in the global economy. Journal of Economic Perspectives, 12(4), 31-50.

Granger, C. W. J. \& Newbold, P. (1986). Forecasting Economic Time Series. Economic Theory and Mathematical Economics (segunda edición). Academic Press.

Haberler, G. (1936). The Theory of International Trade. Londres: William Hodge \& Company.

Heckscher, E. (1935). Mercantilism. Londres: Allen \& Unwin.

Krugman, P. (2000). Technology, trade and factor prices. Journal of International Economics, 50(1), 51-71.

MacKinnon, J. G. (1996). Numerical distribution functions for unit root and cointegration tests. Journey of Applied Econometrics, 11(6), 601-618. 
Mill, S. (1920[1848]). Principles of Political Economy with some of their Applications to Social Philosophy. Londres: Longmans, Green and Co.

Nurkse, R. (1961). Equilibrium and Growth in the World Economy. G. Haberler \& R. M. Stern (eds.). Cambridge: Harvard University Press.

Phillips, P. C. B. \& Perron, P. (1988). Testing for a unit root in time series regression. Biometrika, 75(2), 335-346.

Ricardo, D. (1948[1817]). On the Principles of Political Economy and Taxation. Londres: J. M. Dent and Sons.

Samuelson, P. (1962). The gains from international trade once again. Economic Journal, 72(288), 820-829.

Schwarz, G. (1978). Estimating the dimension of a model. The Annals of Statistics, 6(2), 461-464.

Smith, A. (2012 [1776]). Investigación sobre la naturaleza y causas de la riqueza de las naciones. Madrid: Fondo de Cultura Económica.

Viner, J. (1937). Studies in the Theory of International Trade. Nueva York: Harper and Brothers.

World Bank Group. (2017). Commodity Markets Outlook. Recuperado de http://pubdocs.worldbank.org/ en/743431507927822505/CMO-October-2017-FullReport.pdf 